\title{
Protective effects of ghrelin against oxidative stress, inducible nitric oxide synthase and inflammation in a mouse model of myocardial ischemia/reperfusion injury via the HMGB1 and TLR4/NF-кB pathway
}

\author{
NING SUN ${ }^{1}$, HUI WANG ${ }^{1}$ and LIN WANG $^{2}$ \\ ${ }^{1}$ Department of Geriatrics, Tianjin Medical University General Hospital, Tianjin Geriatrics Institute, Tianjin 300052; \\ ${ }^{2}$ Department of Geriatrics, The Second Hospital of Tianjin Medical University, Tianjin 300211, P.R. China
}

Received July 24, 2015; Accepted June 20, 2016

DOI: $10.3892 / \mathrm{mmr} .2016 .5535$

\begin{abstract}
The present study aimed to investigate the protective effects of ghrelin against oxidative stress, inducible nitric oxide synthase (iNOS) and inflammation in a mouse model of myocardial ischemia/reperfusion injury (MIRI). In addition, the study aimed to determine its underlying mechanisms. A mouse model of MIRI was used in vivo, in order to ascertain the protective effects of ghrelin on MIRI. Commercial kits were used to measure the serum levels of creatine kinase (CK) and lactate dehydrogenase (LDH) in MIRI mice. Furthermore, Evan's Blue-triphenyltetrazolium chloride solution was used to analyze the protective effects of ghrelin against infarct size in MIRI mice. The underlying mechanisms were determined by measuring MIRI-induced tumor necrosis factor (TNF)- $\alpha$, interleukin (IL)-6, superoxide dismutase (SOD), glutathione (GSH), GSH-peroxidase (GSH-PX), malondialdehyde (MDA) and caspase-3/caspase- 9 activities, and iNOS, high mobility group box 1 (HMGB1), Toll-like receptor 4 (TLR4) and nuclear factor $(\mathrm{NF})-\kappa \mathrm{B}$ protein expression in MIRI mice. The results demonstrated that MIRI led to an increase in infarct size; $C K$, LDH, TNF- $\alpha$, IL-6, MDA, caspase-3 and caspase-9 serum levels; and iNOS protein expression. MIRI resulted in inhibition of SOD, FSH and GSH-PX levels. Conversely, these alterations were significantly inhibited following treatment with ghrelin. In addition, the protective effects of ghrelin against MIRI suppressed HMGB1, TLR4 and NF- $\mathrm{NB}$ protein expression in MIRI mice. The present study revealed that ghrelin exerted protective effects against oxidative stress, iNOS and inflammation in MIRI mice via the HMGB1/TLR4/NF- $\mathrm{BB}$ pathway.
\end{abstract}

Correspondence to: Dr Ning Sun, Department of Geriatrics, Tianjin Medical University General Hospital, Tianjin Geriatrics Institute, 154 Anshan Road, Heping, Tianjin 300052, P.R. China E-mail: ningsunnns@126.com

Key words: ghrelin, myocardial ischemia/reperfusion injury, high mobility group box 1 , Toll-like receptor 4 , nuclear factor- $\kappa \mathrm{B}$

\section{Introduction}

With the wide application of intravenous thrombolysis, percutaneous coronary intervention and coronary artery bypass grafting, physicians and researchers are beginning to realize the importance of myocardial ischemia/reperfusion injury (MIRI) (1). The most important therapeutic principle regarding ischemic heart disease and acute myocardial infarction is to open the related blood vessels as early as possible, in order to recover perfusion of ischemic myocardium and rescue myocardial tissue from necrosis (2). However, reperfusion therapy is a double-edged sword, which may aggravate tissue damage at the same time as recovering coronary blood flow (3). Issues including cardiac insufficiency, arrhythmia and extended myocardial infarction may occur; therefore, it is important to identify effective measures for the alleviation of MIRI.

MIRI is a complex pathological process. Well-defined pathogenic mechanisms associated with MIRI include oxidative stress injury, intracellular calcium overload, cell apoptosis, loss of cellular energy, and activation of the inflammatory response via neutrophils (4). Furthermore, MIRI is associated with the activation and inactivation of various regulatory mechanisms and signal pathways.

High mobility group box 1 (HMGB1) is a highly conserved DNA-binding nonhistone protein, which exists in the karyon of eukaryotic cells (5). It has previously been demonstrated that HMGB1 participates in inflammation, where it acts as a proinflammatory cytokine (6). Under inflammatory conditions, HMGB1 is passively released or actively secreted from affected monocytes/macrophages into the extracellular environment (6). Consequently, it can combine with receptor for advanced glycation end products or Toll-like receptors (TLRs). Finally, nuclear factor (NF)- $\kappa \mathrm{B}$ can be activated and the inflammatory response mediated (7).

Ghrelin is found in the stomachs of mice, and was the first endogenous ligand of growth hormone secretagogue receptor to be reported to possess biological activity. Ghrelin is synthesized in the stomach and can promote the secretion of gastric acid and growth hormones via paracrine, autocrine and internal secretion (8). In addition, the functions of ghrelin 
include improving ingestion, gastric acid secretion and gastrointestinal motility, and protecting the mucous membrane of the digestive system (9). Previous studies have reported that ghrelin exerts anti-inflammatory, antioxidative and neuroprotective effects (10-12). Therefore, the present study aimed to determine the protective effects of ghrelin against oxidative stress, inducible nitric oxide synthase (iNOS) and inflammation in MIRI mice, and to investigate the mechanisms underlying the cardioprotective effects of ghrelin.

\section{Materials and methods}

Experimental animals, grouping and MIRI model. A total of 30 male C57BL/6 mice (weight, 20-25 g; age, 6-8 weeks) were obtained from the Shanghai SLAC Laboratory Animal Center Co., Ltd. (Shanghai, China) and were maintained in a standard animal room (temperature, $25 \pm 1^{\circ} \mathrm{C}$; humidity, $70-80 \%$; $12-\mathrm{h}$ light/dark cycle). All mice received humane care in accordance with the Guide for the Care and Use of Laboratory Animals published by the National Institutes of Health (Bethesda, MD, USA) and the present study was approved by the ethics committee of Tianjin Medical University General Hospital. All mice were randomly distributed into three groups of 10 mice: (i) Control group, mice were anesthetized and a suture was passed under the left anterior descending artery without occlusion; (ii) MIRI group, mice were anesthetized and used to generate a MIRI model, the mice were pretreated with $0.5 \mathrm{ml}$ normal saline administered by gavage; (iii) MIRI + ghrelin (Sigma-Aldrich, St. Louis, MO, USA) group, MIRI mice were pretreated with $0.8 \mathrm{mg} / \mathrm{kg}$ ghrelin twice daily by gavage for 3 days.

Briefly, mice were intraperitoneally injected with $7 \mathrm{mg} / \mathrm{kg}$ ketamine and $45 \mathrm{mg} / \mathrm{kg}$ pentobarbital sodium. The left anterior descending coronary artery was ligated and sutured using 7-0 silk. Visual observation of pale color development in the myocardium was considered to indicate regional ischemia. Mice in the control group were also anesthetized and a suture was passed under the left anterior descending artery without occlusion. Mice were sacrificed by overdose of pentobarbital sodium (100 mg/kg). Blood samples were collected from the cardiopuncture of each mouse, and the heart tissues were harvested and washed with ice-cold normal saline.

Serum creatine kinase $(C K)$ and lactate dehydrogenase $(\mathrm{LDH})$ levels. The CK and LDH serum levels were measured in each mouse to determine the degree of myocardial injury using commercial kits (Wuhan Elabscience Biotechnology Co., Ltd., Wuhan, China), according to the manufacturers' protocols.

Assessment of myocardial infarct size. Following $2 \mathrm{~h}$ reperfusion, the left anterior descending artery was re-occluded and $0.3 \mathrm{ml}$ Evan's Blue-triphenyltetrazolium chloride (TTC, $2 \%$ ) was injected into the right jugular vein to determine myocardial infarct size and identify the area prone to ischemic damage. Subsequently, the heart was rapidly excised and rinsed in normal saline, and the left ventricle was isolated and frozen at $-80^{\circ} \mathrm{C}$ for $10-15 \mathrm{~min}$. The frozen left ventricle was cut into $1-\mathrm{mm}$ slices, and was incubated in $1 \%$ TTC for $15 \mathrm{~min}$ at $37^{\circ} \mathrm{C}$. The infarct areas were measured using an image analyzer.
Assessment of caspase activity. Left ventricular samples were homogenized in radioimmunoprecipitation assay lysis buffer (Beyotime Institute of Biotechnology, Nanjing, China) followed by centrifugation at $12,000 \mathrm{x}$ g for $30 \mathrm{~min}$ at $4^{\circ} \mathrm{C}$. The supernatant was collected and used to measure protein concentration with the bicinchoninic acid method. Lysate proteins $(100 \mu \mathrm{g})$ were treated with $50 \mu \mathrm{l}$ Ac-DEVD-pNA and Ac-LEHD-pNA (Beyotime Institute of Biotechnology) for $1 \mathrm{~h}$ at $37^{\circ} \mathrm{C}$. Caspase-3 and caspase- 9 activities were monitored using a microplate reader at $405 \mathrm{~nm}$.

Assessment of tumor necrosis factor (TNF)- $\alpha$, interleukin (IL)-6, superoxide dismutase (SOD), glutathione (GSH), GSH-peroxidase (GSH-PX) and malondialdehyde (MDA) activities. Whole blood samples were homogenized by centrifugation at $12,000 \mathrm{xg}$ for $10 \mathrm{~min}$ at $4^{\circ} \mathrm{C}$. The samples were used to measured TNF- $\alpha$, IL-6, SOD, GSH, GSH-PX and MDA activities in the mice according to the manufacturer's protocols (Beyotime Institute of Biotechnology).

Western blotting. Protein extracts from the heart samples were subjected to western blotting. Briefly, the heart samples were homogenized by centrifugation at $12,000 \mathrm{x} \mathrm{g}$ for $10 \mathrm{~min}$ at $4^{\circ} \mathrm{C}$ in Nuclear and Cytoplasmic Protein Extraction kit (Beyotime Institute of Biotechnology). Protein concentrations were measured using a Bicinchoninic Acid Protein Assay kit (PerkinElmer, Waltham, MA, USA). Equal amounts of protein $(50-80 \mu \mathrm{g})$ were separated by $8-10 \%$ sodium dodecyl sulfate-polyacrylamide gel electrophoresis (Beyotime Institute of Biotechnology) and were transferred to polyvinylidene fluoride membranes.

The membranes were then blocked with $5 \%$ skim milk solution, followed by an overnight incubation at $4{ }^{\circ} \mathrm{C}$ with rabbit anti-HMGB1 (1:4,000; Cell Signaling Technology, Inc., Danvers, MA, USA; cat. no. 6893), rabbit anti-TLR4 (1:3,000; Cell Signaling Technology, Inc.; cat. no. 14358), rabbit anti-NF- $\mathrm{B}$ (1:3,000; Beyotime Institute of Biotechnology; cat. no. AN365) and mouse anti-glyceraldehyde 3-phosphate dehydrogenase (GAPDH; 1:5,000; Beyotime Institute of Biotechnology; cat. no. AF0006). The membranes were then incubated with goat anti-mouse immunoglobulin (Ig)G (1:5,000; Beyotime Institute of Biotechnology; cat. no. A0216) and goat anti-rabbit $\operatorname{IgG}(1: 5,000$; Beyotime Institute of Biotechnology; cat. no. A0208) secondary antibodies for $1 \mathrm{~h}$ at room temperature and washed with Tris-buffered saline/ $0.1 \%$ Tween-20. The relative intensity of the bands was quantified using ImageJ 3.0 software (imagej.nih.gov).

Statistical analysis. Data are presented as the mean \pm standard deviation. Statistical comparisons for continuous variables were performed using analysis of variance and Tukey's test with SPSS 12.0 (SPSS, Inc., Chicago, IL, USA). P<0.05 was considered to indicate a statistically significant difference.

\section{Results}

Protective effects of ghrelin against $C K$ and $L D H$ levels in MIRI mice. As shown in Fig. 1, ghrelin exerted protective effects against CK and LDH levels in MIRI mice. Compared with the control group, MIRI induced a significant increase 
A

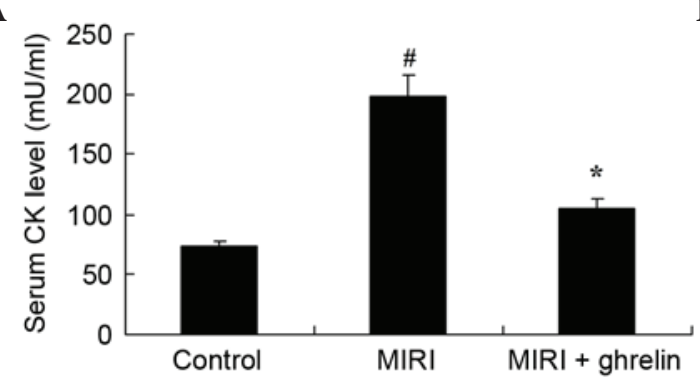

B

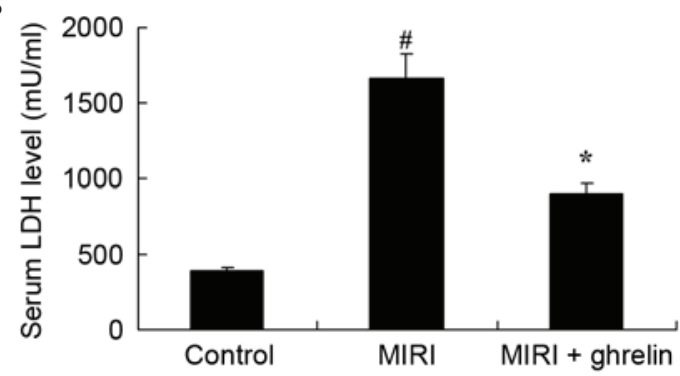

Figure 1. Protective effects of ghrelin against (A) creatine kinase (CK) and (B) lactate dehydrogenase (LDH) levels in myocardial ischemia/reperfusion injury (MIRI) mice. Data are presented as the mean \pm standard deviation. ${ }^{~} \mathrm{P}<0.01$ compared with the control group, ${ }^{*} \mathrm{P}<0.01$ compared with the MIRI group.

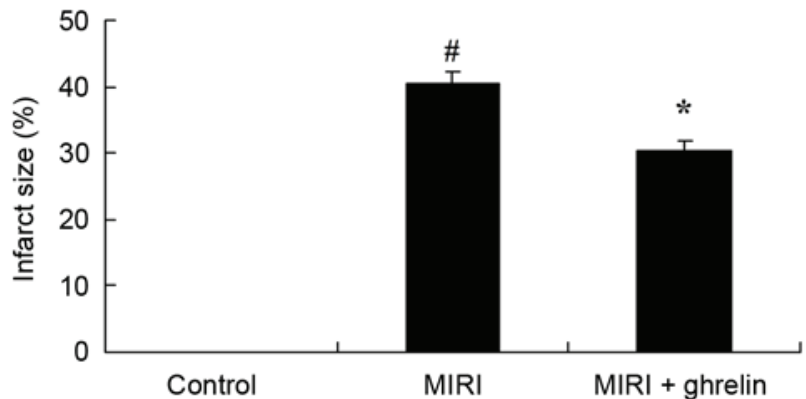

Figure 2. Protective effects of ghrelin against infarct size in myocardial ischemia/reperfusion injury (MIRI) mice. Data are presented as the mean \pm standard deviation. ${ }^{*} \mathrm{P}<0.01$ compared with the control group, ${ }^{*} \mathrm{P}<0.01$ compared with the MIRI group.

in CK levels (Fig. 1A). Furthermore, the LDH levels were markedly increased in the MIRI group compared with in the control mice (Fig. 1B). Conversely, CK and LDH levels were markedly reduced following ghrelin treatment compared with in the MIRI group (Fig. 1A and B).

Protective effects of ghrelin against infarct size in MIRI mice. The effects of ghrelin on representative myocardial infarct size in MIRI mice are presented in Fig. 2. Compared with the control group, MIRI increased myocardial infarct size (Fig. 2). Conversely, ghrelin treatment effectively suppressed myocardial infarct size compared with in the MIRI group (Fig. 2).

Protective effects of ghrelin against caspase-3/caspase-9 activities in MIRI mice. Apoptosis is the major mechanism of cell death following MIRI; therefore, in the present study, caspase-3/caspase-9 activities were measured using a microplate reader. The effects of ghrelin treatment on caspase-3/caspase-9 activities in MIRI mice are presented in Fig. 3. Compared with the control group, MIRI increased caspase-3/caspase- 9 activities. Conversely, pretreatment with ghrelin effectively attenuated the increase in caspase-3/caspase-9 activities in MIRI mice.

Protective effects of ghrelin against oxidative stress in MIRI mice. A microplate reader was used after 3 days to determine the effects of ghrelin on oxidative stress following MIRI. MDA activity levels were significantly higher in the MIRI group compared with in the control mice (Fig. 4). However, SOD, GSH and GSH-PX activities were significantly lower in the MIRI group compared with in the control mice (Fig. 4).
Compared with the MIRI group, MDA levels were markedly decreased, whereas SOD, GSH and GSH-PX activities were markedly increased following treatment with ghrelin (Fig. 4).

Protective effects of ghrelin against iNOS in MIRI mice. The protein expression levels of iNOS following MIRI were detected by western blotting (Fig. 5). Compared with the control group, the protein expression levels of iNOS were significantly increased in response to MIRI. Pretreatment with ghrelin significantly alleviated MIRI-induced iNOS protein expression.

Protective effects of ghrelin against inflammation in MIRI mice. The extent of inflammation was determined to assess the protective effects of ghrelin against MIRI. TNF- $\alpha$ and IL- 6 activities were markedly increased in the MIRI group compared with in the control group (Fig. 6). However, ghrelin treatment significantly inhibited the increase in TNF- $\alpha$ and IL-6 activities in MIRI mice (Fig. 6).

Protective effects of ghrelin on HMGBI in MIRI mice. MIRI induced HMGB1 expression (Fig. 7). Compared with in the control group, HMGB1 protein expression levels were significantly increased in the MIRI group. However, ghrelin pretreatment significantly inhibited the MIRI-induced increase in HMGB1 protein expression (Fig. 7).

Protective effects of ghrelin on TLR4 in MIRI mice. The present study examined the protective effects of ghrelin on TLR4 in MIRI mice. MIRI induced an increase in TLR4 protein expression levels compared with in the control group (Fig. 8). Conversely, ghrelin pretreatment inhibited TLR4 protein expression in MIRI mice (Fig. 8).

Protective effects of ghrelin on $N F-\kappa B$ in MIRI mice. In order to investigate whether ghrelin pretreatment protected against MIRI-induced NF- $\kappa B$, the expression levels of NF- $\kappa B$ were detected. MIRI markedly increased NF- $\mathrm{KB}$ protein expression compared with in the control group (Fig. 9). Following ghrelin treatment, MIRI-induced NF- $\mathrm{BB}$ protein expression was downregulated (Fig. 9).

\section{Discussion}

MIRI refers to serious damage to previously ischemic myocardial tissues after a short period of myocardial blood flow 

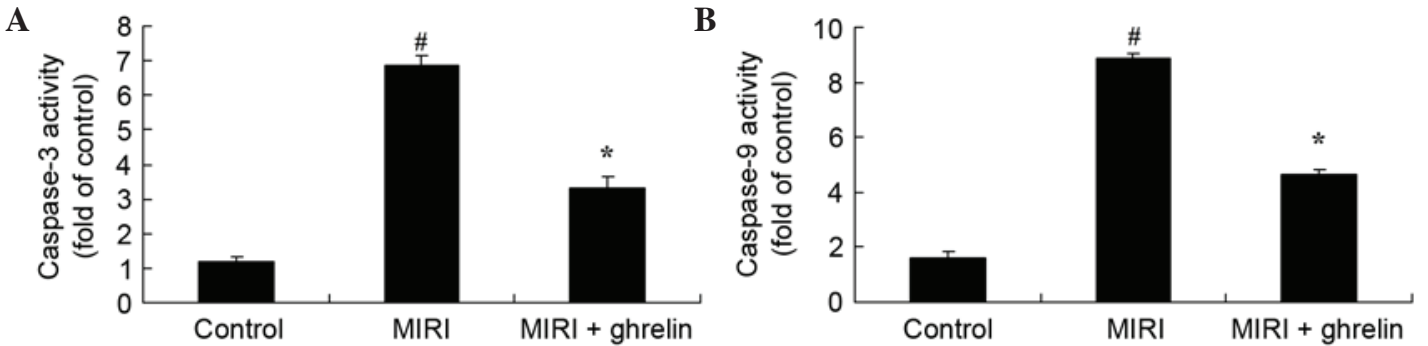

Figure 3. Protective effects of ghrelin against (A) caspase-3 and (B) caspase-9 activities in myocardial ischemia/reperfusion injury (MIRI) mice. Data are presented as the mean \pm standard deviation. ${ }^{~} \mathrm{P}<0.01$ compared with the control group, $\mathrm{P}<0.01$ compared with the MIRI group.
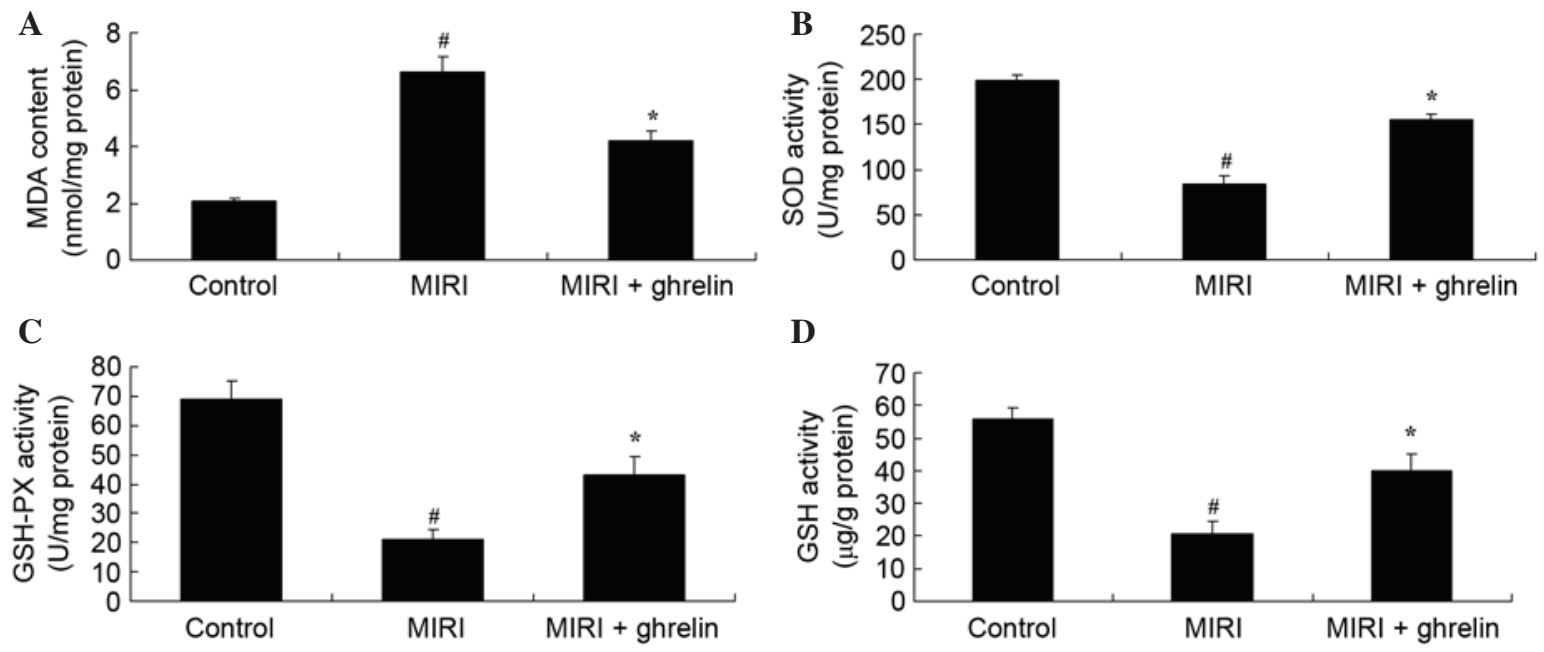

D

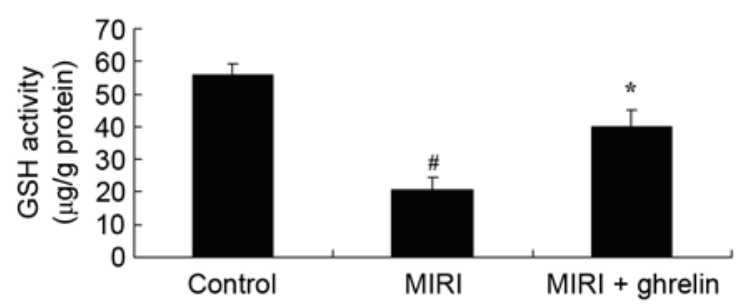

Figure 4. Protective effects of ghrelin against oxidative stress in myocardial ischemia/reperfusion injury (MIRI) mice. Protective effect of ghrelin against (A) malondialdehyde (MDA), (B) superoxide dismutase (SOD), (C) glutathione-peroxidase (GSH-PX) and (D) GSH activities in MIRI mice. Data are presented as the mean \pm standard deviation. ${ }^{*} \mathrm{P}<0.01$ compared with the control group, ${ }^{*} \mathrm{P}<0.01$ compared with the MIRI group.

A

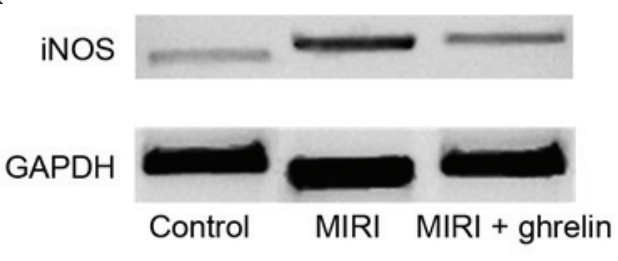

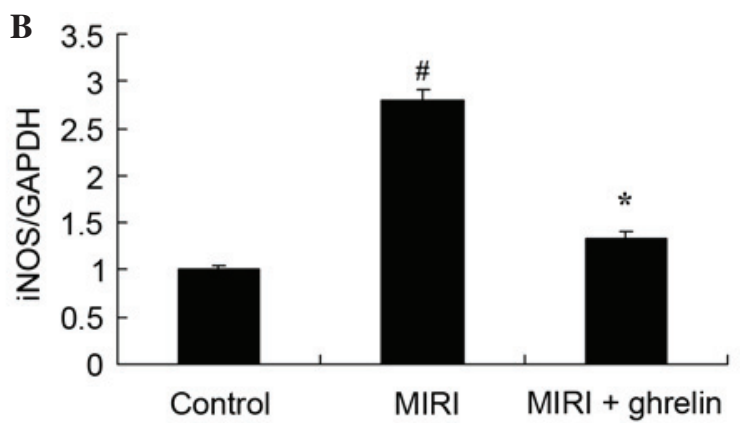

Figure 5. Protective effects of ghrelin against inducible nitric oxide synthase (iNOS) in myocardial ischemia/reperfusion injury (MIRI) mice were detected by (A) western blotting and (B) statistical analysis of iNOS protein expression. Data are presented as the mean \pm standard deviation. ${ }^{\#} \mathrm{P}<0.01$ compared with the control group, ${ }^{*} \mathrm{P}<0.01$ compared with the MIRI group. GAPDH, glyceraldehyde 3-phosphate dehydrogenase.

failure (13). The clinical manifestations of MIRI after recanalization of an obstructed coronary artery include reperfusion arrhythmias, extension of the area of infarcted cardiac muscle, cardiac insufficiency or sudden mortality (14). Post-reperfusion of ischemic myocardium, metabolic disturbances to myocardial cells and morphological structural damage are also serious complications (15). In addition, the number of necrotic myocardial cells is increased by $\sim 25 \%$ (16). Therefore, methods of coronary artery recanalization, including thrombolysis, are a double-edged sword (17), since MIRI may lead to novel complications, which greatly decrease the efficacy of therapies attempting to improve myocardial blood supply and rescue infarcted myocardium. The present study demonstrated that the protective effects of ghrelin inhibited MIRI-induce CK and LDH levels, and reduced infarct size in a mouse model of MIRI.

HMGBl shares features with cytokines, and can be actively secreted by activated immune cells and act on the surface receptors of immune cells and endothelial cells in order to induce expression of inflammatory factors and further HMGB1 release, resulting in promotion of the inflammatory cascade (18). Furthermore, HMGB1 has been reported to 

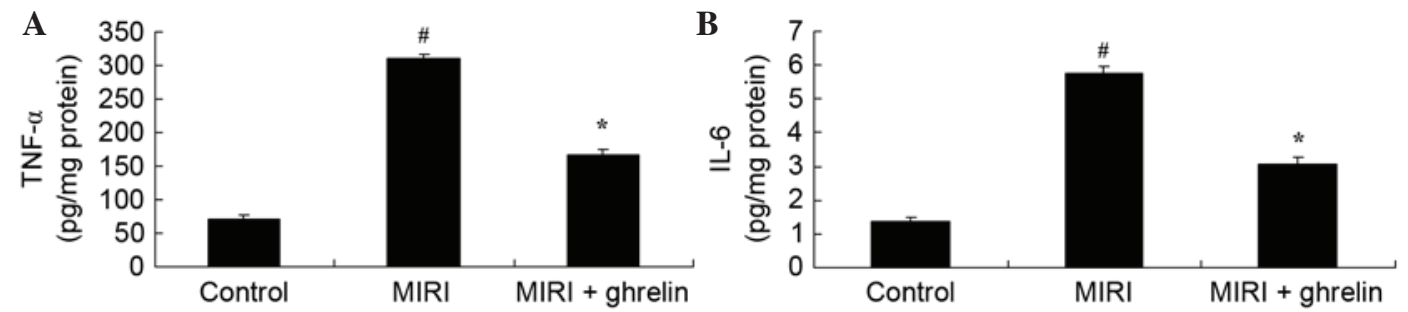

Figure 6. Protective effects of ghrelin against inflammation in myocardial ischemia/reperfusion injury (MIRI) mice. Protective effects of ghrelin against (A) tumor necrosis factor (TNF)- $\alpha$ and (B) interleukin (IL)- 6 activities in MIRI mice. Data are presented as the mean \pm standard deviation. ${ }^{\#} \mathrm{P}<0.01$ compared with the control group, ${ }^{*} \mathrm{P}<0.01$ compared with the MIRI group.
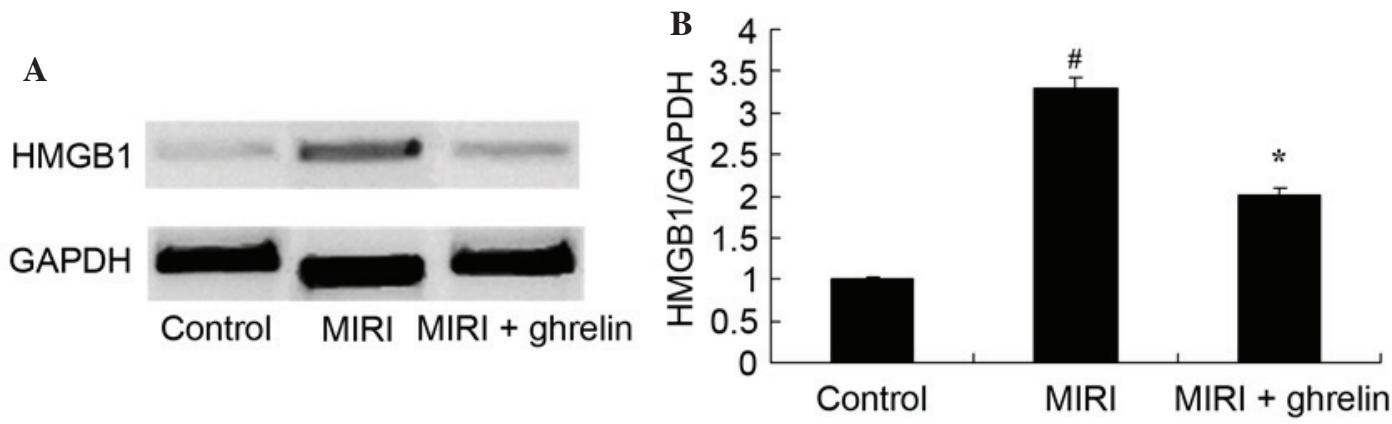

Figure 7. Protective effects of ghrelin on high mobility group box 1 (HMGB1) in myocardial ischemia/reperfusion injury (MIRI) mice were detected by (A) western blotting and (B) statistical analysis of HMGB1 protein expression. Data are presented as the mean \pm standard deviation. ${ }^{~} \mathrm{P}<0.01$ compared with the control group, ${ }^{\mathrm{P}}<0.01$ compared with the MIRI group. GAPDH, glyceraldehyde 3-phosphate dehydrogenase.

A
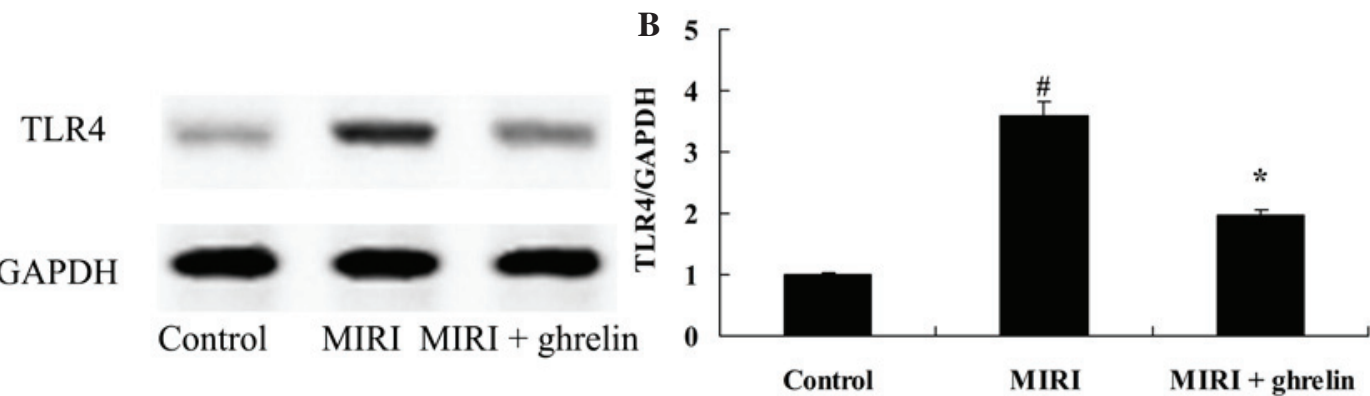

Figure 8. Protective effects of ghrelin on Toll-like receptor 4 (TLR4) in myocardial ischemia/reperfusion injury (MIRI) mice were detected by (A) western blotting and (B) statistical analysis of TLR4 protein expression. Data are presented as the mean \pm standard deviation. ${ }^{\text {} P}<0.01$ compared with the control group, ${ }^{*} \mathrm{P}<0.01$ compared with the MIRI group. GAPDH, glyceraldehyde 3-phosphate dehydrogenase.

stimulate the release of cytokines and adhesion molecules; promote inflammatory cells; destroy epithelial barriers and extend the inflammatory response (19). The results of the present study suggested that ghrelin pretreatment inhibited caspase- 3 and caspase- 9 activities; alleviated oxidative stress, iNOS protein expression and inflammation; and suppressed HMGB1 protein expression in MIRI mice. Wang et al (20) demonstrated that ghrelin protects mice against endotoxemia-induced acute kidney injury via the suppression of nitric oxide, and inhibition of TNF- $\alpha$, IL- $1 \beta$ and IL- 6 . Koyuturk et al (10) indicated that ghrelin inhibited apoptosis, cell proliferation and the oxidant-antioxidant system via caspase- 8 and caspase- 3 in the liver of neonatal diabetic rats. Furthermore, Ercan et al (21) suggested that ghrelin treatment suppressed sodium metabisulfite-induced oxidative stress and caspase-3 expression in rat gastric mucosa.
TLRs are receptors of HMGB1. TLRs are signal transduction transmembrane receptors that are found on the cell surface. Among the TLR family, TLR2/4 are able to recognize the majority of pathogenic microorganisms (22). Distributed in mononuclear cells, macrophages, neutrophils, dendritic cells and cancer cells, TLR2/4 exerts innate immune functions against bacteria, viruses, fungi and other pathogenic infections (23). It acts as a bridge between congenital immunity and acquired immunity (24). TLR 2 and TLR4 can integrate with HMGB1, which is secreted by macrophages and neutrophils, and induce the occurrence of inflammation (23). In addition, it has been reported that TLR 2 and TLR 4 are potential receptors of HMGB1. The present study indicated that ghrelin treatment inhibited TLR4 protein expression in MIRI mice. Liu et al (25) reported that ghrelin reduced high glucose-induced $\mathrm{PC} 12$ cell apoptosis via the TLR4/NF-кB pathway. 
A

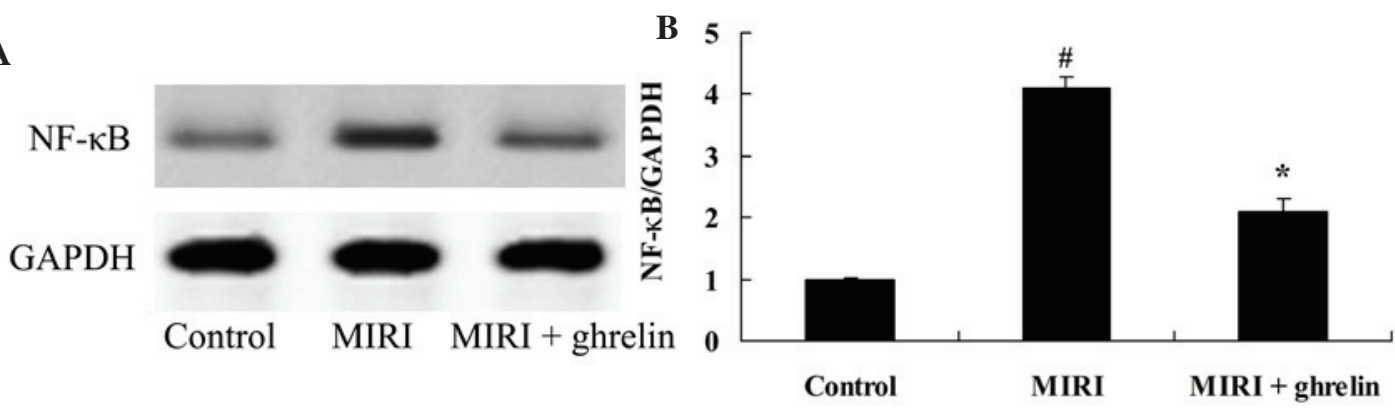

Figure 9. Protective effects of ghrelin on nuclear factor (NF)-кB in myocardial ischemia/reperfusion injury (MIRI) mice as detected by (A) western blotting and (B) statistical analysis of NF- $\mathrm{KB}$ protein expression. Data are presented as the mean \pm standard deviation. "P<0.01 compared with the control group, ${ }^{*} \mathrm{P}<0.01$ compared with the MIRI group. GAPDH, glyceraldehyde 3-phosphate dehydrogenase.

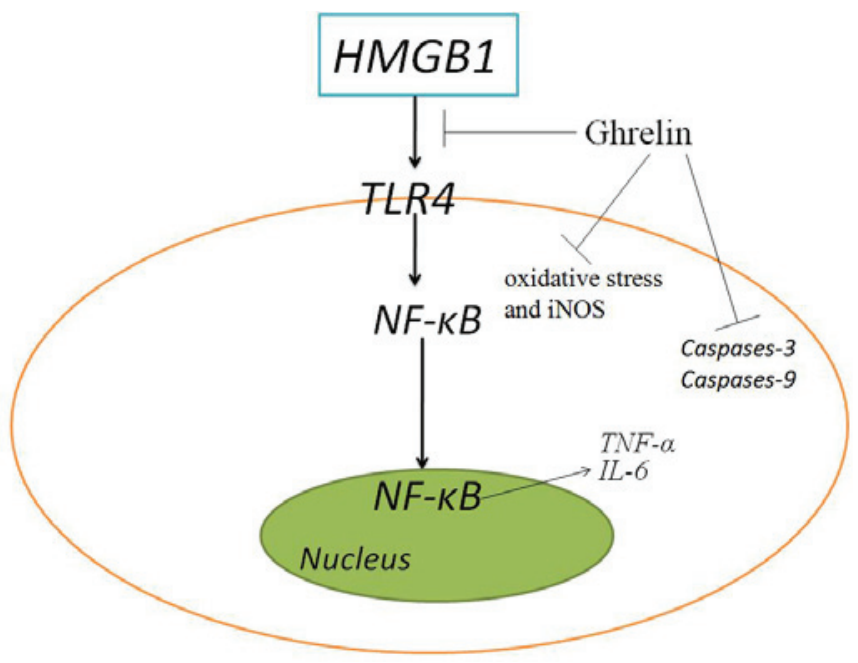

Figure 10. Protective effects of ghrelin against oxidative stress, inducible nitric oxide synthase (iNOS) and inflammation in myocardial ischemia/reperfusion injury mice via the high mobility group box 1 (HMGB1) and Toll-like receptor 4 (TLR4)/nuclear factor (NF)- $\mathrm{KB}$ pathway. TNF- $\alpha$, tumor necrosis factor- $\alpha$; IL-6, interleukin-6; SOD, superoxide dismutase; GSH, glutathione; GSH-PX, GSH-peroxidase; MDA, malondialdehyde.

Following integration of cytokines and receptor proteins, a series of signal transduction molecules are required to transmit signals into cells. The signal transduction pathway mediated by TLR $2 / 4$ is as follows. Specific ligands that are recognized by the TLR2/4 transmembrane domain induce the release of signals into the cells (26). Subsequently, the toll-IL-1 receptor cytoplasmic domain of TLR2/4 combines with various downstream molecular regulators, thus activating downstream signal transduction cascades by MyD88-dependent or -independent pathways (27). Finally, transcription factors, such as NF- $\kappa \mathrm{B}$ and interferon regulatory factor 3 , are activated; inflammatory cytokines, such as IFN- $\beta$, and thus inflammation are induced; inflammatory cell infiltration is induced by chemotaxis, and innate immunity is activated (28). The present study revealed the protective effects of ghrelin, which was shown to inhibit $\mathrm{NF}-\kappa \mathrm{B}$ expression in MIRI mice. Liu et al (25) reported that ghrelin reduced high glucose-induced PC12 cell apoptosis via the TLR4/NF- $\kappa$ B pathway. Furthermore, Rezaeian et al (29) demonstrated that ghrelin protects musculocutaneous tissue via $\mathrm{NF}-\kappa \mathrm{B}$ in ischemic necrosis.

In conclusion, as shown in Fig. 10, ghrelin exerts protective effects against oxidative stress, iNOS and inflammation in MIRI mice via the HMGB1 andTLR4/NF- $\kappa \mathrm{B}$ pathway. Therefore, ghrelin may be a clinically useful agent in the treatment of MIRI.

\section{Acknowledgements}

The present study was supported the by Tianjin Municipal Health Bureau Science Foundation (grant no. 2011KE113) and the Tianjin Municipal Natural Science Foundation (grant no. 13JCYBJC36400).

\section{References}

1. Garcia Gómez-Heras S, Alvarez-Ayuso L, Torralba Arranz A and Fernandez-Garcia H: Purkinje fibers after myocardial ischemia-reperfusion. Histol Histopathol 30: 841-853, 2015.

2. Smiley D, Smith MA, Carreira V, Jiang M, Koch SE, Kelley M, Rubinstein J, Jones WK and Tranter M: Increased fibrosis and progression to heart failure in MRL mice following ischemia/reperfusion injury. Cardiovasc Pathol 23: 327-334, 2014.

3. Zhao J, Wang F, Zhang Y, Jiao L, Lau WB, Wang L, Liu B, Gao E, Koch WJ, Ma XL and Wang Y: Sevoflurane preconditioning attenuates myocardial ischemia/reperfusion injury via caveolin-3-dependent cyclooxygenase-2 inhibition. Circulation 128 (Suppl 1): S121-S129, 2013.

4. Xue L, Wu Z, Ji XP, Gao XQ and Guo YH: Effect and mechanism of salvianolic acid $\mathrm{B}$ on the myocardial ischemia-reperfusion injury in rats. Asian Pac J Trop Med 7: 280-284, 2014.

5. Park SY, Lee SW, Kim HY, Lee WS, Hong KW and Kim CD: HMGB1 induces angiogenesis in rheumatoid arthritis via HIF-1 $\alpha$ activation. Eur J Immunol 45: 1216-1227, 2015.

6. Kornblit B, Munthe-Fog L, Madsen HO, Strom J, Vindelov L and Garred P: Association of HMGB1 polymorphisms with outcome in patients with systemic inflammatory response syndrome. Crit Care 12: R83, 2008.

7. Ibrahim ZA, Armour CL, Phipps S and Sukkar MB: RAGE and TLRs: Relatives, friends or neighbours? Mol Immunol 56: 739-744, 2013.

8. Muller TD, Nogueiras R, Andermann ML, Andrews ZB, Anker SD, Argente J, Batterham RL, Benoit SC, Bowers CY, Broglio F, et al: Ghrelin. Mol Metab 4: 437-460, 2015.

9. Jonsson E: The role of ghrelin in energy balance regulation in fish. Gen Comp Endocrinol 187: 79-85, 2013.

10. Koyuturk M, Sacan O, Karabulut S, Turk N, Bolkent S, Yanardag R and Bolkent S: The role of ghrelin on apoptosis, cell proliferation and oxidant-antioxidant system in the liver of neonatal diabetic rats. Cell Biol Int 39: 834-841, 2015.

11. Huang CX, Yuan MJ, Huang H, Wu G, Liu Y, Yu SB, Li HT and Wang T: Ghrelin inhibits post-infarct myocardial remodeling and improves cardiac function through anti-inflammation effect. Peptides 30: 2286-2291, 2009.

12. Matsumoto A, Yamafuji M, Tachibana T, Nakabeppu Y, Noda M and Nakaya H: Oral 'hydrogen water' induces neuroprotective ghrelin secretion in mice. Sci Rep 3: 3273, 2013. 
13. Wang J, Yang H, Hu X, Fu W, Xie J, Zhou X, Xu W and Jiang H: Dobutamine-mediated heme oxygenase-1 induction via PI3K and p38 MAPK inhibits high mobility group box 1 protein release and attenuates rat myocardial ischemia/reperfusion injury in vivo. J Surg Res 183: 509-516, 2013.

14. King AL and Lefer DJ: Cytoprotective actions of hydrogen sulfide in ischaemia-reperfusion injury. Exp Physiol 96: 840-846, 2011.

15. Ekeløf S, Rosenberg J, Jensen JS and Gögenur I: Pharmacological attenuation of myocardial reperfusion injury in a closed-chest porcine model: A systematic review. J Cardiovasc Transl Res 7: 570-580, 2014.

16. Ruiz-Meana M and Garcia-Dorado D: Translational cardiovascular medicine (II). Pathophysiology of ischemia-reperfusion injury: New therapeutic options for acute myocardial infarction. Rev Esp Cardiol 62: 199-209, 2009.

17. Quintana M, Kahan T and Hjemdahl P: Pharmacological prevention of reperfusion injury in acute myocardial infarction. A potential role for adenosine as a therapeutic agent. Am J Cardiovasc Drugs 4: 159-167, 2004.

18. Ramasamy R, Yan SF and Schmidt AM: Stopping the prima RAGE reaction in myocardial infarction: Capturing adaptive responses to heal the heart? Circulation 117: 3165-3167, 2008.

19. Wang Q, Cheng Y, Xue FS, Yuan YJ, Xiong J, Li RP, Liao X and Liu JH: Postconditioning with vagal stimulation attenuates local and systemic inflammatory responses to myocardial ischemia reperfusion injury in rats. Inflamm Res 61: 1273-1282, 2012.

20. Wang W, Bansal S, Falk S, Ljubanovic D and Schrier R: Ghrelin protects mice against endotoxemia-induced acute kidney injury. Am J Physiol Renal Physiol 297: F1032-F1037, 2009.

21. Ercan S, Basaranlar G, Gungor NE, Kencebay C, Sahin P, Celik-Ozenci C and Derin N: Ghrelin inhibits sodium metabisulfite induced oxidative stress and apoptosis in rat gastric mucosa. Food Chem Toxicol 56: 154-161, 2013.
22. Herzog C, Lorenz A, Gillmann HJ, Chowdhury A, Larmann J, Harendza T, Echtermeyer F, Müller M, Schmitz M, Stypmann J, et al: Thrombomodulin's lectin-like domain reduces myocardial damage by interfering with HMGB1-mediated TLR2 signalling. Cardiovasc Res 101: 400-410, 2014.

23. Kwak MS, Lim M, Lee YJ, Lee HS, Kim YH, Youn JH, Choi JE and Shin JS: HMGB1 binds to lipoteichoic acid and enhances TNF- $\alpha$ and IL- 6 Production through HMGB1-Mediated Transfer of Lipoteichoic Acid to CD14 and TLR2. J Innate Immun 7: 405-416, 2015.

24. Liu Q, Wang J, Liang Q, Wang D, Luo Y, Li J, Janicki JS and Fan D: Sparstolonin B attenuates hy poxia-reoxygenation-induced cardiomyocyte inflammation. Exp Biol Med (Maywood) 239: 376-384, 2014.

25. Liu X, Xiao Q, Zhao K and Gao Y: Ghrelin inhibits high glucose-induced PC12 cell apoptosis by regulating TLR4/NF-kappaB pathway. Inflammation 36: 1286-1294, 2013.

26. Song R, Ao L, Zhao KS, Zheng D, Venardos N, Fullerton DA and Meng X: Soluble biglycan induces the production of ICAM-1 and MCP-1 in human aortic valve interstitial cells through TLR2/4 and the ERK1/2 pathway. Inflamm Res 63: 703-710, 2014.

27. Hao H, Gufu H, Lei F, Dang L and Zhongliang Y: Baicalin suppresses expression of TLR $2 / 4$ and $N F-\kappa B$ in chlamydia trachomatis-infected mice. Immunopharmacol Immunotoxicol 34: 89-94, 2012.

28. Tu XK, Yang WZ, Chen JP, Chen Y, Ouyang LQ, Xu YC and Shi SS: Curcumin inhibits TLR2/4-NF-kB signaling pathway and attenuates brain damage in permanent focal cerebral ischemia in rats. Inflammation 37: 1544-1551, 2014.

29. Rezaeian F, Wettstein R, Scheuer C, Bäumker K, Bächle A, Vollmar B, Menger MD and Harder Y: Ghrelin protects musculocutaneous tissue from ischemic necrosis by improving microvascular perfusion. Am J Physiol Heart Circ Physiol 302: H603-H610, 2012. 